\title{
Time series analysis of poison control data
}

\author{
Dan Giurca, MD
}

$\mathrm{T}$ he US Poison Control Centers' National Poison Data System (NPDS) publishes annual reports describing exposures to various substances among the general population. ${ }^{1}$ Table 22B of each NPDS report shows the number of outcomes from exposures to different pharmacologic treatments in the United States, including psychotropic medications. ${ }^{2}$ In this Table, the relative morbidity (RM) of a medication is calculated as the ratio of serious outcomes (SO) to single exposures (SE), where $\mathrm{SO}=$ moderate + major + death. In this article, I use the NPDS data to demonstrate how time series analysis of the RM ratios for hypertension and psychiatric medications can help predict $\mathrm{SO}$ associated with these agents, which may help guide clinicians' prescribing decisions. ${ }^{2,3}$

\section{Time series analysis of hypertension medications}

Due to the high prevalence of hypertension, it is not surprising that more suicide deaths occur each year from calcium channel blockers (CCB) than from lithium (37 vs 2, according to 2017 NPDS data). ${ }^{3}$ I used time series analysis to compare SO during 2006-2017 for 5 classes of hypertension medications: $\mathrm{CCB}$, beta blockers (BB), angiotensin-converting enzyme inhibitors (ACEI), angiotensin receptor blockers (ARB), and diuretics (Figure 1, page e6).

Time series analysis of 2006-2017 data predicted the following number of deaths for 2018: CCB $\geq 33$, BB $\geq 17$, ACEI $\leq 2$, $A R B$, and diuretics $\leq 1$. The observed deaths in 2018 were 41, 23, 0, 0, and 1, respectively. ${ }^{2}$ The 2018 predicted RM were CCB $10.66 \%$, BB $11.10 \%$, ACEI $3.51 \%$,
ARB 2.04\%, and diuretics 3.38\%. The 2018 observed RM for these medications were $11.01 \%, 11.37 \%, 3.02 \%, 2.40 \%$, and $2.88 \%$, respectively. ${ }^{2}$

Because the NPDS data for hypertension medications was only provided by class, in order to detect differences within each class, I used the relative lethality (RL) equation: $\mathrm{RL}=310 x$ / LD50, where $x$ is the maximum daily dose of a medication prescribed for 30 days, and LD50 is the rat oral lethal dose 50. The RL equation represents the ratio of a 30-day supply of medication to the human equivalent LD50 for a $60-\mathrm{kg}$ person. ${ }^{4}$ The RL equation is useful for comparing the safety of various medications, and can help clinicians avoid prescribing a lethal amount of a given medication (Figure 2, page e 7 ). For example, the equation shows that among CCB, felodipine is 466 times safer than verapamil and 101 times safer than diltiazem. Not surprisingly, 20062018 data shows many deaths via intentional verapamil or diltiazem overdose vs only 1 reference to felodipine. A regression model shows significant correlation and causality between RL and SO over

\section{Every issue of CURRent PsychiatRY} has its 'Pearls'

\section{Yours could be found here.}

Read the 'Pearls' guidelines for manuscript submission at MDedge.com/ CurrentPsychiatry/page/pearls. Then, share with your peers a 'Pearl' of wisdom from your practice.
Dr. Giurca is a Locum Tenens Psychiatrist, Office of Mental Health, New York.

\section{Disclosure}

The author reports no financial relationships with any companies whose products are mentioned in this article, or with manufacturers of competing products. 
More suicide

deaths occur each year from calcium channel blockers than from lithium

\section{Figure 1}

\section{Time series analysis of the relative morbidity of hypertension medications}

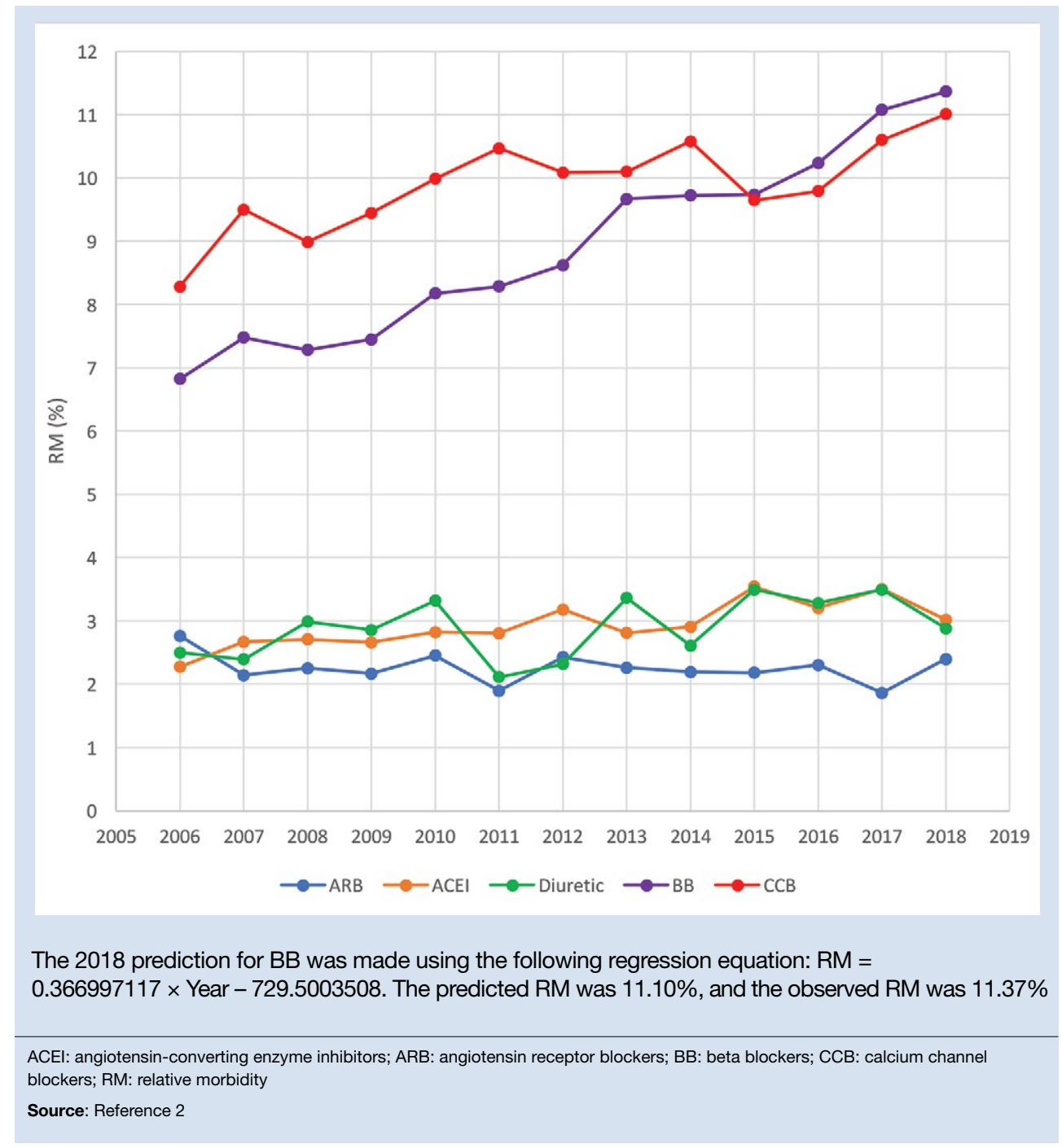

time. $^{5}$ Integrating all 3 mathematical models suggests that the higher RM of CCB and BB may be caused by the high RL of verapamil, diltiazem, nicardipine, propranolol, and labetalol.

These mathematical models can help physicians consider whether to switch the patient's current medication to another class with a lower RM. For patients who need a BB or CCB, prescribing a medication with a lower RL within the same class may be another option. The data suggest that avoiding hypertension medications with RL >100\% may significantly decrease morbidity and mortality.

\section{Predicting serious outcomes of psychiatric medications}

The 2018 NPDS data for psychiatric medications show similarly important results. ${ }^{2}$ For example, the lithium RM is predictable over time (Figure 3, page e8) and has been consistently the highest among psychiatric medications. Using 2006-2017 NPDS data, ${ }^{3}$ I predicted that the 2018 lithium RM would be $41.56 \%$. The 2018 observed 


\section{Relative lethality of hypertension medications $\mathrm{s}^{\mathrm{a}}$}

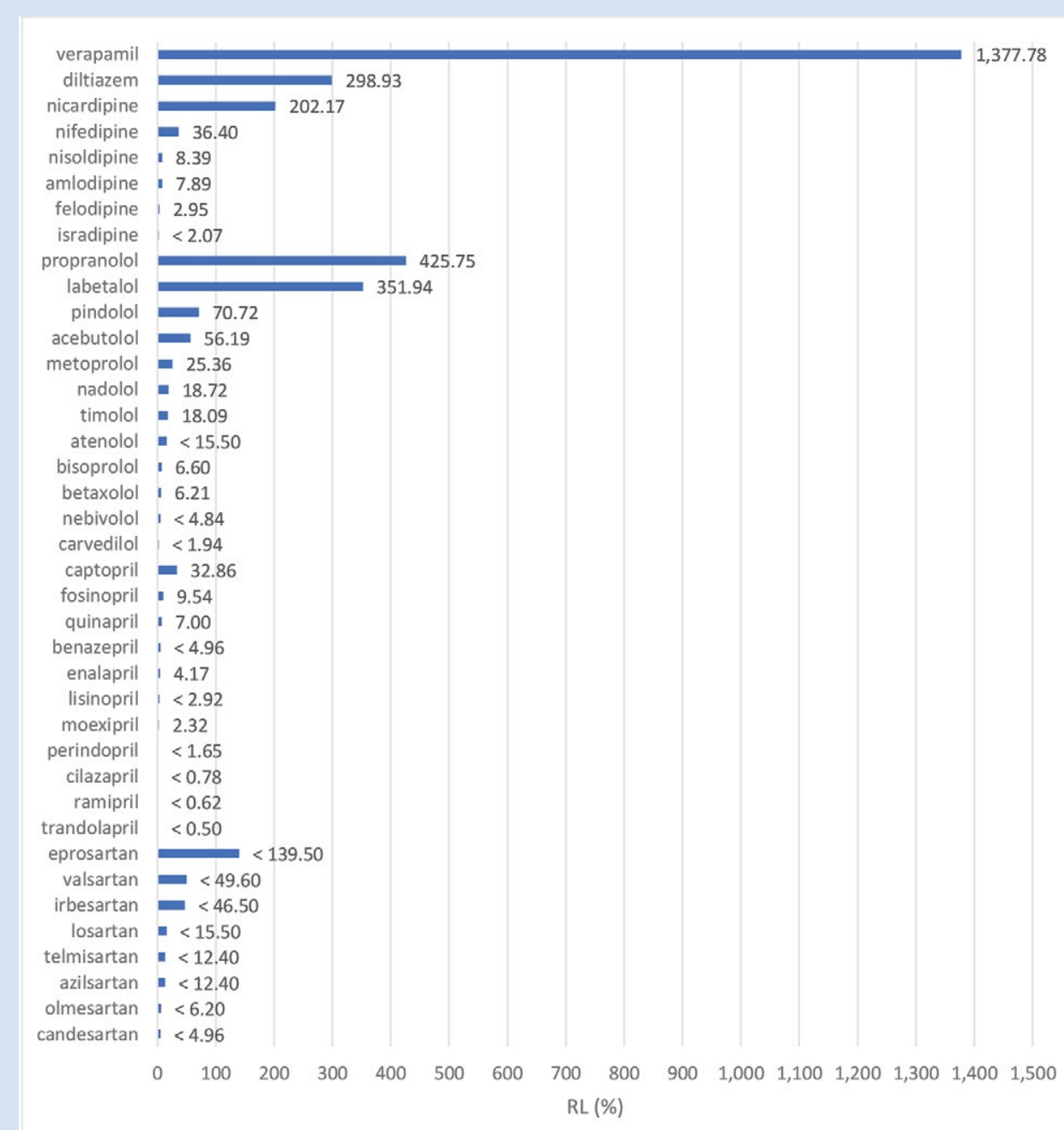

${ }^{\mathrm{a}} \mathrm{RL}=($ dose $\times 30$ days $) /($ LD50 $\times 60 \mathrm{~kg} / 6.2)$

If the medication is prescribed at half the maximum daily dose, the $R L$ is decreased by a factor of 2 . If a 1-day supply is given, the $R L$ is decreased by a factor of 30 , but the ratio of one medication to another would remain the same

LD50: lethal dose 50; RL: relative lethality

lithium RM was $41.45 \% .^{2}$ I created a linear regression model for each NPDS report from 2013 to 2018 to illustrate the correlation between RL and adjusted SO for 13 psychiatric medications. ${ }^{2,3,6,7}$ To account for different sample sizes among medications, the lithium SE for each respective year was used for all medications (adjusted SO $=\mathrm{SE} \times \mathrm{RM})$. A time series analysis of these regression models shows that $\mathrm{SO}$ data points hover in the same $y$-axis region from year to year, with a corresponding RL on the $x$-axis: escitalopram $6.33 \%$, citalopram $15.50 \%$, mirtazapine $28.47 \%$, paroxetine $37.35 \%$, sertraline $46.72 \%$, fluoxetine $54.87 \%$, venlafaxine $99.64 \%$, duloxetine $133.33 \%$, trazodone $269.57 \%$, bupropion $289.42 \%$, amitriptyline $387.50 \%$, doxepin $632.65 \%$, and lithium $1062.86 \%$ (Figure 4, page e 8 ). Every year, the scatter plot shape

\section{The relative}

lethality equation can help clinicians avoid prescribing a lethal amount of a given medication 
Lithium's relative morbidity has been consistently the highest among psychiatric medications

\section{Figure 3}

Time series analysis of lithium relative morbidity

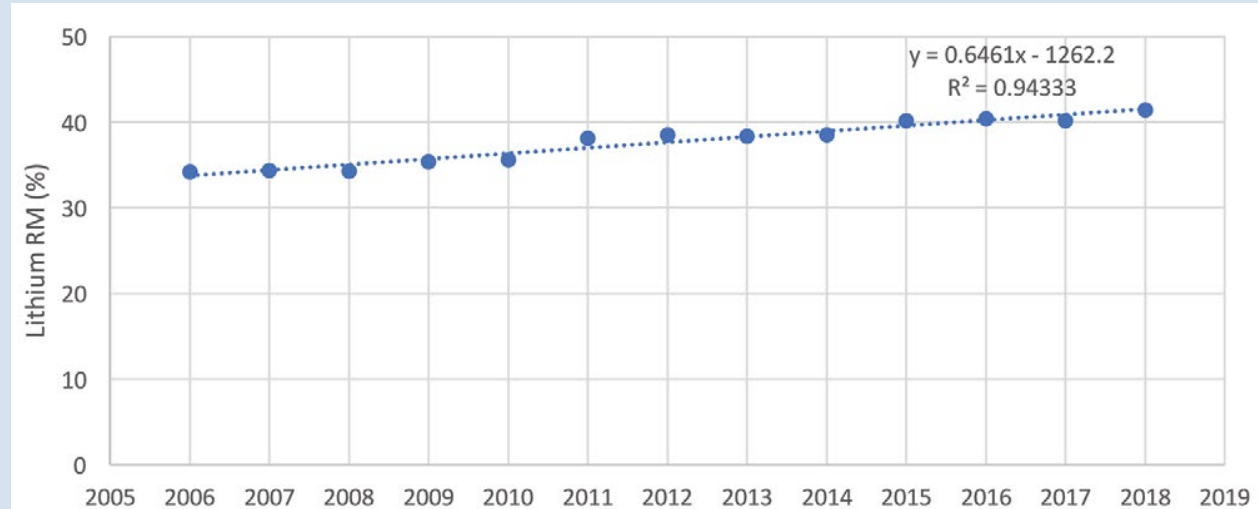

The 2018 prediction was made using the following regression equation: $\mathrm{RM}=0.649630694 \times$ Year -1269.397514 . The predicted RM was $41.56 \%$, and the observed RM was $41.45 \%$. The 2019 and 2020 predictions are $42.21 \%$ and $42.86 \%$, respectively

RM: relative morbidity

\section{Figure 4}

Time series analysis of linear regression models for 13 psychiatric medications
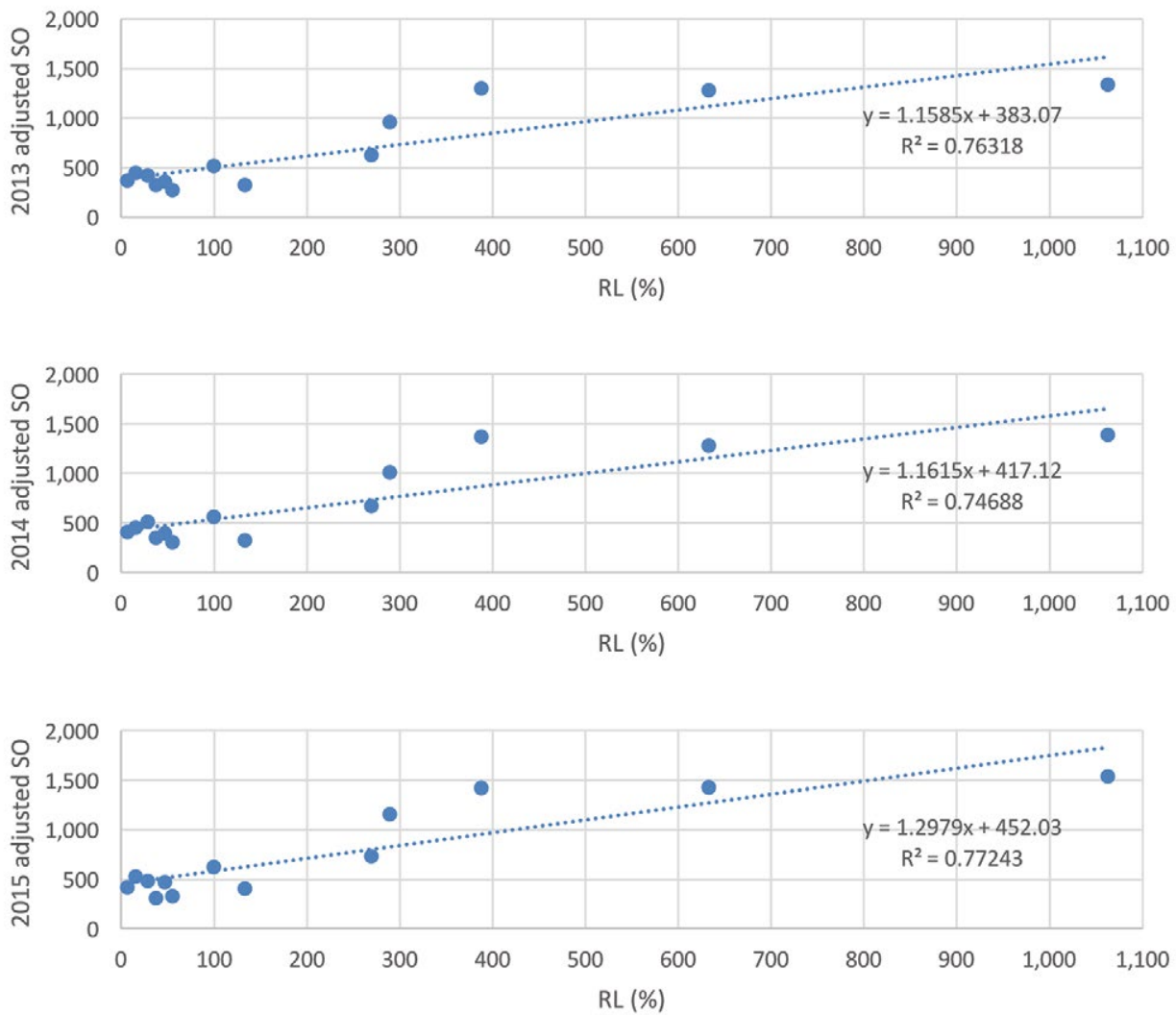
remains approximately the same, which suggests that both $\mathrm{SO}$ and RM can be predicted over time. Medications with RL $>300 \%$ have $\mathrm{SO} \approx 1500(\mathrm{RM} \approx 40 \%)$, and those with $\mathrm{RL}<100 \%$ have $\mathrm{SO} \approx 500$ ( $\mathrm{RM} \approx 13 \%$ ).

Time series analysis of NPDS data sheds light on hidden patterns. It may help clinicians discern patterns of potential SO associated with various hypertension and psychiatric medications. RL based on rat experimental data is highly correlated to RM based on human observational data, and the causality is self-evident. On a global scale, data-driven prescribing of medications with RL $<100 \%$ could potentially help prevent millions of SO every year.

\section{References}

1. National Poison Data System Annual Reports. American Association of Poison Control Centers. https://www.aapcc. org/annual-reports. Updated November 2019. Accessed May 5, 2020.

2. Gummin DD, Mowry JB, Spyker DA, et al. 2018 Annual Report of the American Association of Poison Control Centers' National Poison Data System (NPDS): 36th Annual Report. Clin Toxicol (Phila). 2019;57(12):1220-1413.

3. Gummin DD, Mowry JB, Spyker DA, et al. 2017 Annual Report of the American Association of Poison Control Centers' National Poison Data System (NPDS): 35th Annual Report. Clin Toxicol (Phila). 2018;56(12):1213-1415.

4. Giurca D. Decreasing suicide risk with math. Current Psychiatry. 2018;17(2):57-59,A,B.

5. Giurca D. Data-driven prescribing. Current Psychiatry. 2018;17(10):e6-e8.

6. Mowry JB, Spyker DA, Brooks DE, et al. 2015 Annual Report of the American Association of Poison Control Centers' National Poison Data System (NPDS): 33rd Annual Report. Clin Toxicol (Phila). 2016;54(10):924-1109.

7. Gummin DD, Mowry JB, Spyker DA, et al. 2016 Annual Report of the American Association of Poison Control Centers' National Poison Data System (NPDS): 34th Annual Report. Clin Toxicol (Phila). 2017;55(10):1072-1252.
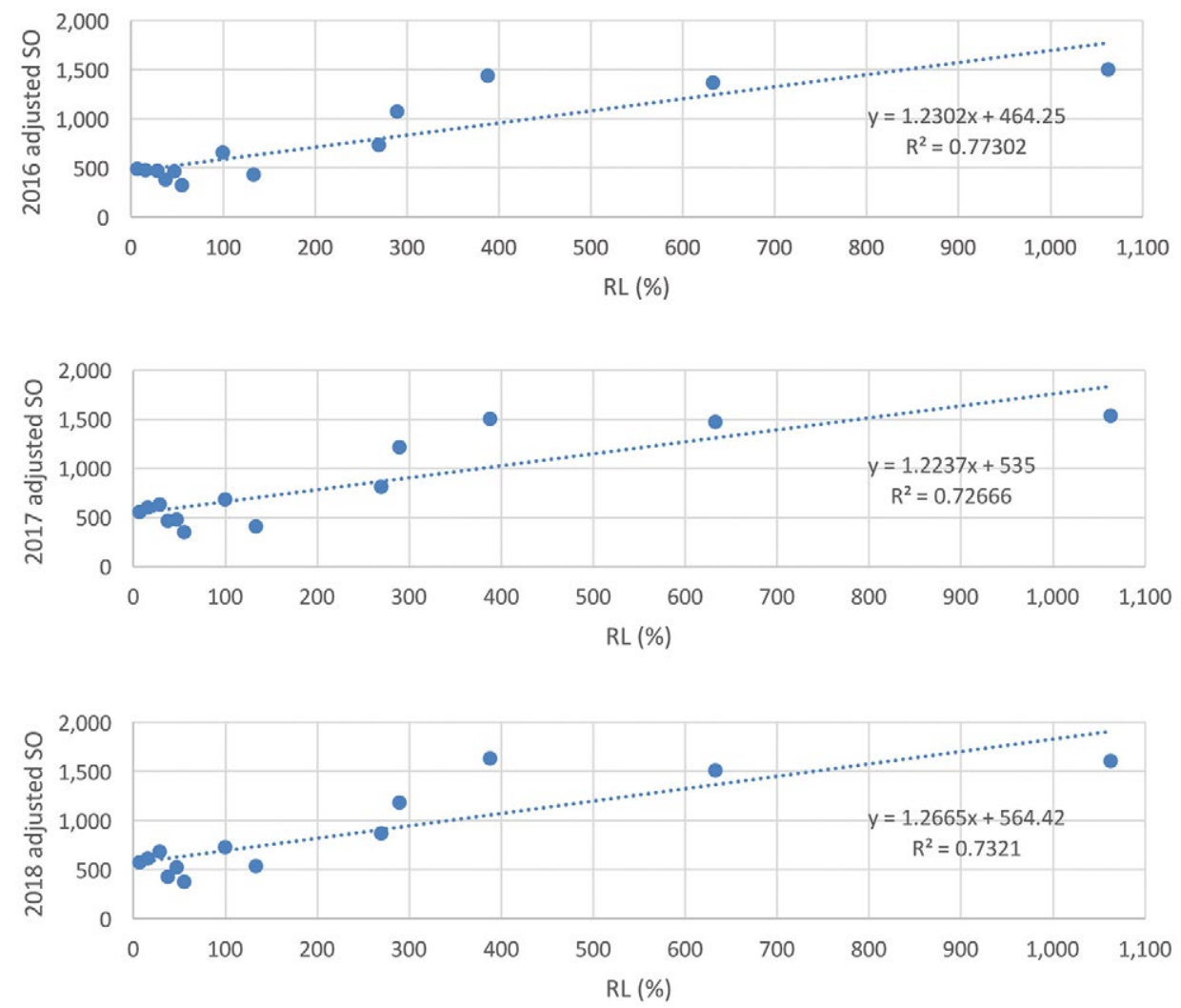